\title{
15 years of follow up of non-affective functional psychotic disorders showed a high risk of relapse, suicide, and chronicity
}

Wiersma D, Nienhuis FJ, Slooff CJ, et al. Natural course of schizophrenic disorders: a 15-year followup of a Dutch incidence cohort. Schizophr Bull 1998 Jan;24:75-85.

\section{Question}

What is the natural course of schizophrenia and other non-affective functional psychoses and which factors at onset predict the course?

\section{Design}

Inception cohort followed up for 15 years.

\section{Setting}

2 provinces in the north of the Netherlands.

\section{Patients}

82 patients between 15 and 44 years of age $(61 \%<25$ y, $52 \%$ men) with a first ever onset of functional psychosis of a non-affective type diagnosed using the $I C D$-9. Patients had to have $\geqslant 1$ of the following symptoms: hallucinations, delusions of a nonaffective type, bizarre or grossly inappropriate behaviour, or thought or speech disorder. Exclusion criteria were organic brain disease, severe mental retardation, or alcohol or drug dependence.

\section{Assessment of prognostic factors}

Standardised assessments of psychopathology, psychological impairments, negative symptomatology, social disability, and use of mental healthcare services were used at baseline and follow up. Sociodemographic and premorbid characteristics were also ascertained at baseline.

\section{Main outcome measures}

Remission of psychotic symptoms, chronicity of psychosis, relapses, suicide, and predictors of course.

\section{Main results}

Diagnosis after 6 months: according to DSM-III-R criteria, 63\% had schizophrenia or schizophreniform psychosis, and 30\% had other psychosis; according to ICD-10 criteria, $76 \%$ had schizophrenia or schizophreniform psychosis, and 18\% had other psychosis.* After 15 years of follow up, $27 \%$ of the patients $\{95 \%$ CI $18 \%$ to $38 \%\} \uparrow$ had complete remission, $50 \%\{$ CI $39 \%$ to $61 \%\} \uparrow$ had partial remission (mainly leaving a negative syndrome) after several episodes, $11 \%$ CI 5\% to 20\%\}† were continuously psychotic, and in $12 \%\{$ CI $6 \%$ to $21 \%\} \uparrow$ the course was unknown. $66 \%\{$ CI $55 \%$ to $76 \%\} \uparrow$ of patients had $\geqslant 1$ relapse. After each episode, on average $17 \%$ of patients (range $8-27 \%$ ) did not remit and 21\% (range 15-25\%) remitted only partially with negative symptoms. After remission from the first episode, $43 \%$ relapsed within 1 year, $55 \%$ within 2 years, and $70 \%$ within 5 years. After 15 years, $15 \%$ had not relapsed after recovery from the first episode. 9 patients ( $11 \%$ \{CI $5 \%$ to $50 \%\} \dagger$ ) committed suicide, 4 during their first or second psychotic episode. Cox regression was used to investigate predictors of the time to recovery from first psychotic episode and time to first relapse. Delay in treatment was the only predictor of duration of a first psychotic episode (hazard ratio 2.33, $\mathrm{p}<0.01$ ). There were no significant predictors of time to first relapse.

\section{Conclusion}

15 years of follow up of non-affective functional psychotic disorders showed a high risk of relapse, suicide, and chronicity.

*Information supplied by author.

†Numbers calculated from data in article.

Sources of funding: World Health Organisation; the Laureate Foundation; participating field centres; the Praeventiefonds.

For correspondence: Dr D Wiersma, Department of Social Psychiatry, WHO Collaborating Centre, University of Groningen, PO Box 30.001,9700 RB Groningen, the Netherlands. Fax +31503611 699.

Abstract and commentary also published in Evidence-Based Medicine.

\section{Commentary}

This long term follow up study by Wiersma et al provides valuable data on evolution of symptoms; use of health care; and response to treatment. The broad definition of schizophrenia at inception allowed inclusion of patients who developed core symptoms only later in the course of their illness.

Patients showed above average levels of education and premorbid functioning in an area with well organised and easily accessible mental health care. Patients dependent on alcohol and drugs were excluded. These characteristics of the cohort need to be considered when using the findings clinically because contemporaneous British cohorts of inception ${ }^{1}$ and heterogeneous cases ${ }^{2}$ and recent prevalence studies ${ }^{3}$ all found lower social class of origin and poorer premorbid educational attainment in patients with schizophrenia. In one survey, $11 \%$ of patients were found to be misusing alcohol and $11 \%$ using illicit drugs.

There was a tendency for episodes of illness after the first to be of increasingly long duration ( $47 \%$ of patients developed chronic symptoms). Premorbid functioning is regarded as a guide to severity of illness, ${ }^{4}$ and its failure to predict clinical outcome in this cohort is important. It may be because of an association with social dependence or insidious onset and delay in seeking treatment, all of which were predictive, but this cannot be inferred from the current analysis.

Teifion W Davies, PhD, MBBS, MRCPsych University of London London, UK

1 The Scottish Schizophrenia Research Group.

BrJ Psychiatry 1987;150:331-3.
2 McCreadie RG. BrJPsychiatry 1982;140:582-6.

3 Foster K, Meltzer H, Gill B, et al. OPCS surveys of psychiatric disorder in Great Britain. Report 8. Adults with a psychotic disorder living in the community. London: HMSO, 1996.

4 McCreadie RG, Connolly MA, Williamson DJ, et al. BrJ Psychiatry 1994;165:340-6.

Editor's note: See also DSM-III-R and ICD-10 diagnoses of schizophrenia had higher predictive validity than that of the ICD-9 and CATEGO S + diagnoses [abstract]. EvidenceBased Medicine 1997 Nov-Dec:2:190. Abstract of: Mason P, Harrison G, Croudace T, et al. Br J Psychiatry 1997 Apr;170:321-7. 